\title{
Minimally Invasive Percutaneous Endoscopic Discectomy and Drainage for Infectious Spondylodiscitis
}

\author{
Tsai-Sheng Fu, Lih-Hui Chen, Wen-Jer Chen
}

\begin{abstract}
The primary goals for treating infectious spondylodiscitis are to make an accurate diagnosis, isolate the causative organism, and prescribe effective antibiotic therapy based on the culture data. A positive culture of the responsible organism is not required for diagnosis, although it is extremely important for successful treatment and prevention of further morbidity. Surgical intervention is usually reserved for cases that are unresponsive to antibiotic therapy and for patients who have developed progressive spinal deformity or instability, epidural abscesses, or neurological impairment. However, the incidence of perioperative morbidity is particularly increased in elderly patients or in those with poor general condition. With improved endoscopic instruments and techniques, our clinical experiences demonstrate that spinal infections can be successfully treated by minimally invasive percutaneous endoscopic debridement. Direct endoscopic observation and collection of sufficient quantities of samples for microbiological

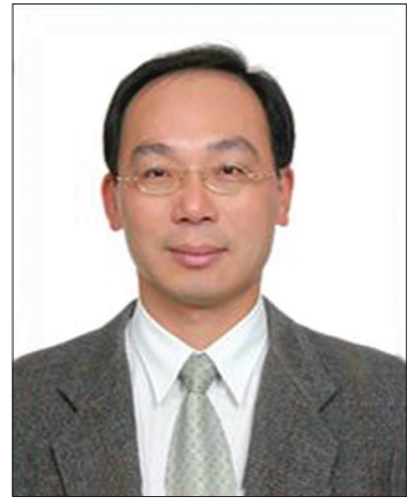

Dr. Tsai-Sheng Fu examinations from the infected region are usually possible. This article summarizes the diagnostic and therapeutic values of percutaneous endoscopic discectomy and drainage (PEDD) used to treat patients with spondylodiscitis. Our clinical evidence-based survey suggests that PEDD can provide adequate retrieval of specimens and has high diagnostic efficacy, thereby enabling prompt and sensitive antibiotic therapy to the offending pathogens. We propose that PEDD is an effective alternative for treating infectious spondylodiscitis and should be considered prior to extensive anterior surgery in selected cases. This method is particularly suitable for patients with early-stage spinal infection or serious medical conditions.
\end{abstract}

(Biomed J 2013;36:168-174)

\section{Key words: infectious spondylodiscitis, minimally invasive spinal surgery, percutaneous drainage, percutaneous endoscopic discectomy, spinal biopsy}

$\mathrm{I}_{\mathrm{n}}^{\mathrm{n}}$ nfectious spondylodiscitis is a major infection of the nucleus pulposus with secondary involvement of the cartilaginous endplate and vertebral bone, which can occur spontaneously in immunocompromised patients due to hematogenous spread from other inflammatory foci or following diagnostic and therapeutic procedures. ${ }^{[1,2]}$ The incidence of spinal infections has been increasing in recent years, which is primarily due to contemporary medical treatment for serious systematic diseases prolonging life expectancy, increasing intravenous drug use, the rising incidence of healthcare-associated infections, and the increased number of immunocompromised and aging individuals. ${ }^{[3]}$
Infectious spondylodiscitis is basically a medical disease and as a rule treated conservatively. The standard conservative treatment includes administration of sensitive antibiotics after proper identification of the causative organisms and external mechanical support. Surgery is only reserved for cases that are unresponsive to conservative therapy. For preventing surgical intervention and perioperative morbidities, early and accurate isolation of the causative organisms for prescribing an effective antibiotic therapy based on the culture data becomes very important.

Strategies for detection of causative organisms include blood and urine cultures and an attempt to obtain tissues

From the Department of Orthopaedic Surgery, Chang Gung Memorial Hospital at Linkou, Chang Gung University College of Medicine, Taoyuan, Taiwan

Received: May. 22, 2012; Accepted: Nov. 26, 2012

Correspondence to: Dr. Tsai-Sheng Fu, Department of Orthopaedic Surgery, Chang Gung Memorial Hospital at Linkou. 5, Fusing St., Gueishan, Taoyuan 333, Taiwan (R.O.C.). Tel: 866-3-3281200; ext: 3612; Fax: 886-3-3278113; E-mail: fts111@adm.cgmh.org.tw

DOI: $10.4103 / 2319-4170.112742$ 
directly from the infected spinal region by image guidance needle biopsy. However, the success rate for bacteriological diagnosis is variable by these methods. Our strategy is to use a minimally invasive endoscopic technique for direct observation and collection of sufficient quantities of samples for microbiological examinations. This article summarizes the diagnostic and therapeutic values of this alternative method used to treat patients with infectious spondylodiscitis.

\section{Diagnosis}

The clinical symptoms of infectious spondylodiscitis are non-specific and include back pain with or without sciatica, fever, and malaise. Diagnosis is based on clinical symptoms, laboratory studies such as elevated erythrocyte sedimentation rate (ESR) and C-reactive protein (CRP) values, and roentgenographic and magnetic resonance image (MRI) findings with confirmation provided by histopathologic examination. The initial radiographic sign of infectious spondylodiscitis is commonly a collapsed intervertebral disc space with or without subtle erosion of the vertebral endplate. In more aggressive cases, osteolysis can result in substantial shortening and collapse of the vertebral bodies, ultimately resulting in instability. ${ }^{[4-6]} \mathrm{A}$ positive culture of the responsible organism is not required for diagnosis, although it is extremely important for successful treatment.

\section{Conventional treatments}

The primary goals for treating of spinal infections are to make an accurate diagnosis, isolate the causative organisms, and prescribe an effective antibiotic therapy based on the culture data. Conservative treatment is adequate in cases involving mild destruction or earlier-stage infection. Surgical intervention is usually reserved for cases that are unresponsive to antibiotic therapy and for patients who have developed progressive spinal deformity or instability, epidural abscesses, or neurological impairment. ${ }^{[6-10]}$ The surgical goals for treating infectious spondylodiscitis include debridement of necrotic bone and surrounding tissue, drainage of associated paraspinal abscesses, and correction of kyphosis with spinal fusion. However, perioperative morbidities increase with anterior debridement or combined anterior and posterior surgery, particularly in elderly patients or in patients with poor general condition. ${ }^{[1]}$ Thus, early diagnosis of the infection and prompt initiation of the appropriate antibiotic therapy against the cultured organisms are crucial to ensure successful treatment of spinal infection and prevent further morbidities. ${ }^{[12-14]}$

\section{Identification of the causative organism}

A microbiological diagnosis is essential to enable an appropriate choice of therapeutic agents. Blood culture is a cost-effective and simple method for identifying the causative organisms in the presence of pyrexia, as the infection typically originates from a hematogenous source ${ }^{[3]}$ Direct needle biopsy from the infectious region may be needed to confirm the diagnosis and to optimize the administration of sensitive antibiotics. Computed tomography (CT)-guided biopsy is the most common procedure for bacteriologic diagnosis; however, needle biopsy for bacteriological diagnosis has been reported to have a variable success rate in patients with spinal infections. ${ }^{[15-21]} \mathrm{An}$ inadequate amount of biopsy tissue from CT-guided biopsy often leads to false-negative cultures and low diagnostic efficacy in detecting the infectious organisms, which often leads surgeons to perform more invasive spinal surgeries. ${ }^{[22]}$ Furthermore, a high degree of radiation exposure during the procedure is another important concern. ${ }^{[23,24]}$

\section{Minimally invasive spinal surgery for spondylodiscitis}

In the orthopedic field, treatment of large joint infections typically includes open arthrotomy, debridement, and antibiotic therapy. ${ }^{[25,26]}$ Gradually, open arthrotomy has been superseded by minimally invasive percutaneous arthroscopic debridement. ${ }^{[27,28]}$ Similarly, several minimally invasive spinal techniques had been developed and used to treat infectious spondylodiscitis. ${ }^{[29-37]} \mathrm{CT}$-guided percutaneous catheter drainage, ${ }^{[19]}$ percutaneous transpedicular discectomy and drainage, ${ }^{[29,32]}$ percutaneous drainage and continuous irrigation, ${ }^{[3,36]}$ percutaneous suction aspiration and drainage, ${ }^{[34,37]}$ and automated percutaneous flexible nucleotome debridement ${ }^{[30,35]}$ through a unilateral or bilateral posterolateral approach have been reported as efficient and safe procedures in the management of early-stage spondylodiscitis. However, these procedures lack intraoperative virtual images monitoring for debridement of the lesion site. Furthermore, the innovations and advances in fiberoptic technology and camera systems have allowed surgeons to view the intervertebral disc and epidural space directly. With improved endoscopic instruments and techniques, more extensive debridement and eradication of the infected tissue can be achieved. As with the treatment of large joint infections, spinal infections can be successfully treated by percutaneous endoscopic debridement.

\section{Minimally invasive percutaneous endoscopic technique for lumbar spondylodiscitis}

Percutaneous endoscopic discectomy (PED) was first employed for treating uncomplicated herniated discs in the early 1980s ${ }^{[38]}$ Recently, numerous minimally invasive percutaneous endoscopic procedures for lumbar disc herniation have been developed. The clinical outcomes of these procedures are comparable to those of conventional open surgery. ${ }^{[38-40]}$ The PED technique has been performed for treating lumbar disc hernia- 
tion at our institution since 2000. The minimal invasiveness and simplicity of the technique led us to apply percutaneous endoscopic discectomy and drainage (PEDD) as a modality for treating earlier-stage infectious spondylodiscitis. ${ }^{[21,41]}$ Direct endoscopic observation and collection of sufficient sample quantities for microbiological examinations from the infected region are possible with this technique. Eradication and debridement of the infected and necrotic tissue from a disc and the epidural space can be achieved under endoscopic monitoring. Moreover, postoperative negative-pressure Hemovac drainage can continuously remove the pathogens and abscesses within the infected area. A combination of good debridement and a full course of sensitive antimicrobial therapy resulted in favorable patient outcomes. This technique, which involves a minimal percutaneous approach, has also been used in the treatment of pyogenic spondylodiscitis in patients with serious comorbidities and in cases of complicated infection with significant abscess formation or destruction of vertebrae. ${ }^{[42]}$

\section{PEDD procedures}

The PEDD procedures are performed via a posterolateral percutaneous approach using the Yeung Endoscopic Spinal System (Richard Wolf GmbH, Knittlingen, Germany) under local anesthesia and conscious sedation. The patient is positioned prone on a radiolucent frame suitable for intraoperative fluoroscopy. The entry point to the target site is determined under fluoroscopic guidance. Sterile preparation and draping are performed, local anesthesia is administered, a spinal needle is inserted directly into the targeted disc, and the abscess is aspirated for microorganism cultures [Figure 1A]. A guide wire is introduced into the disc space through the spinal needle and the spinal needle is subsequently withdrawn. After creating a small stab-wound incision (approximately $1 \mathrm{~cm}$ ), a dilator and a cannulated sleeve are guided over the wire and passed sequentially into the disc space [Figure 1B]. Fluoroscopic examination is performed in two orthogonal planes to verify the correct position of the dilator tip. The tissue dilator is then removed, and the cutting tool is inserted to harvest a biopsy specimen first. Discectomy forceps are inserted through the cannulated sleeve to extract additional tissue from the infected disc under fluoroscopic monitoring [Figure 1C]. The debrided tissues typically contain necrotic disc material and parts of the vertebral endplates of adjacent vertebrae. The specimen is subjected to aerobic and anaerobic cultures, tuberculosis culture, polymerase chain reaction, fungal culture, and histopathologic examinations. After biopsy and debridement, irrigation is performed using normal saline and the intradiscal lesion is endoscopically examined [Figure 1D]. Finally, a drainage tube (diameter, $3.2 \mathrm{~mm}$ ) is inserted into the debrided disc space [Figure $2 \mathrm{~A}]$ and connected to a negative-pressure pump (Hemovac; Zimmer, Dover, OH, USA) [Figure 2B]. All tubes are left in place until the drainage is stopped or reduced to less than $10 \mathrm{ml} /$ day for three consecutive days. After the

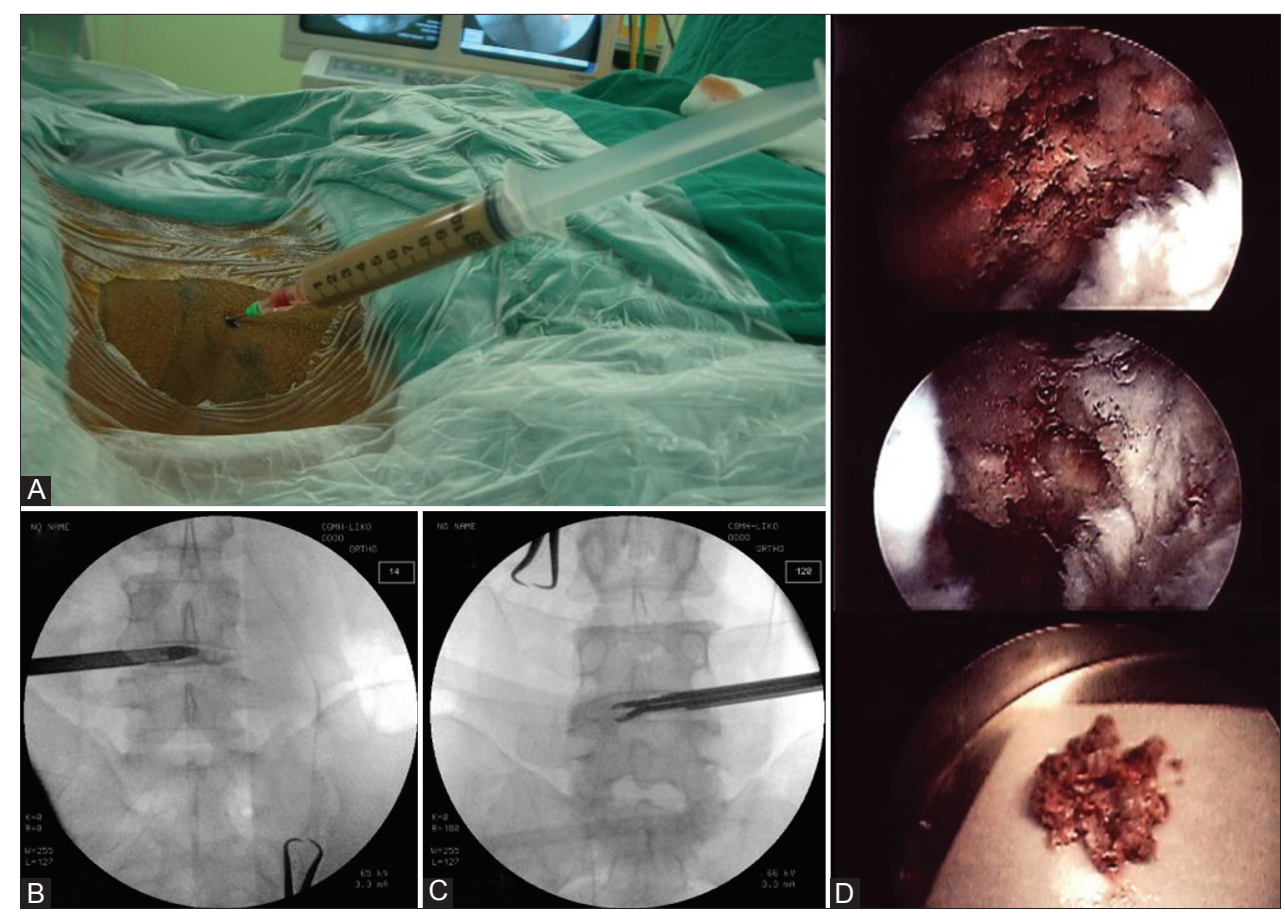

Figure 1: Percutaneous endoscopic discectomy and drainage (PEDD) procedures. (A) The spinal needle is inserted directly into the targeted disc and the abscess is aspirated. (B) The dilator and cannulated sleeve are guided into the disc space as the working channel. (C) The forceps is inserted through the cannulated sleeve to extract tissue from the infected disc under fluoroscopic monitoring. (D) Endoscopic findings of the destructed intervertebral disc and the extract tissue for bacteriological and histopathologic examinations. 
operation, the patients are allowed to walk while wearing a Taylor's brace.

\section{Diagnostic and therapeutic value of PEDD versus CT-guided biopsy}

CT-guided needle biopsy is reported to have a variable success rate for bacteriological diagnosis in patients with spinal infections. ${ }^{[15-21]}$ Fouquet et al., used a Mazabraud trocar for biopsy and obtained bacteriological diagnoses

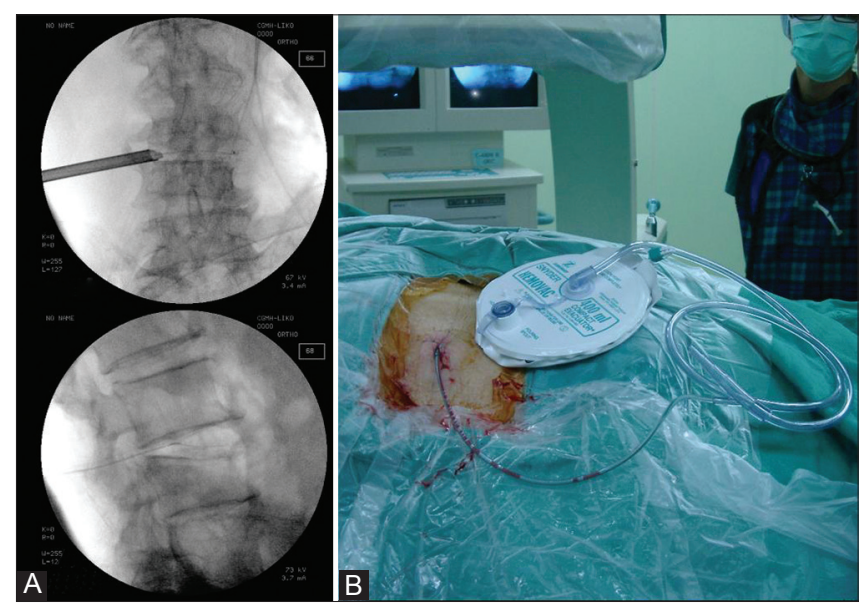

Figure 2: A negative-pressure (Hemovac) drainage tube is inserted within the infected area for continuous removal of the pathogens and abscesses. (A) Anteroposterior and lateral fluoroscopic images verify the correct position of the drainage tube tip within the debrided disc space. (B) A negative-pressure pump (Hemovac) is connected to the drainage tube. in $9(36 \%)$ of 25 patients. ${ }^{[16]}$ Rankine et al., analyzed 20 patients undergoing percutaneous spinal biopsies for spinal infections. The organisms were isolated in 6 of 12 patients not taking antibiotics, whereas the organisms were isolated in only 2 of 8 patients taking antibiotics. ${ }^{[18]}$ Staatz et al., performed CT-guided percutaneous catheter drainage and reported $16(76 \%)$ positive cultures in 21 patients. ${ }^{[19]}$

We enrolled patients who underwent PEDD and CT-guided biopsy for identifying the offending pathogens and compared the diagnostic and therapeutic values of these methods. ${ }^{[21]}$ Twenty patients were treated with PEDD and the other 32 patients underwent CT-guided biopsies. In the PEDD group, causative bacteria were identified in 18 (90\%) of 20 biopsy specimens, whereas in the CT-guided biopsy group, causative bacteria were identified in $15(47 \%)$ of 32 specimens. Five patients (25\%) in the PEDD group underwent anterior fusion surgery with autografting due to progressive infection or kyphotic instability. Of the 15 patients with positive culture findings in the CT-guided biopsy group, 8(53\%) underwent surgical treatment. Of the remaining 17 patients with negative biopsies, 10 (59\%) patients required surgical treatment. No biopsy-related complications or side effects were observed in either group. In comparison with CT-guided biopsy, PEDD provided retrieval of greater number of specimens, yielding higher diagnostic efficacy. Therefore, the rate of secondary surgical intervention was reduced after debridement of infected tissues by PEDD, combined with prompt application of adequate antimicrobial therapy. The positive-culture rate of PEDD is comparable with those obtained with open biopsy. ${ }^{[7]}$ We propose that

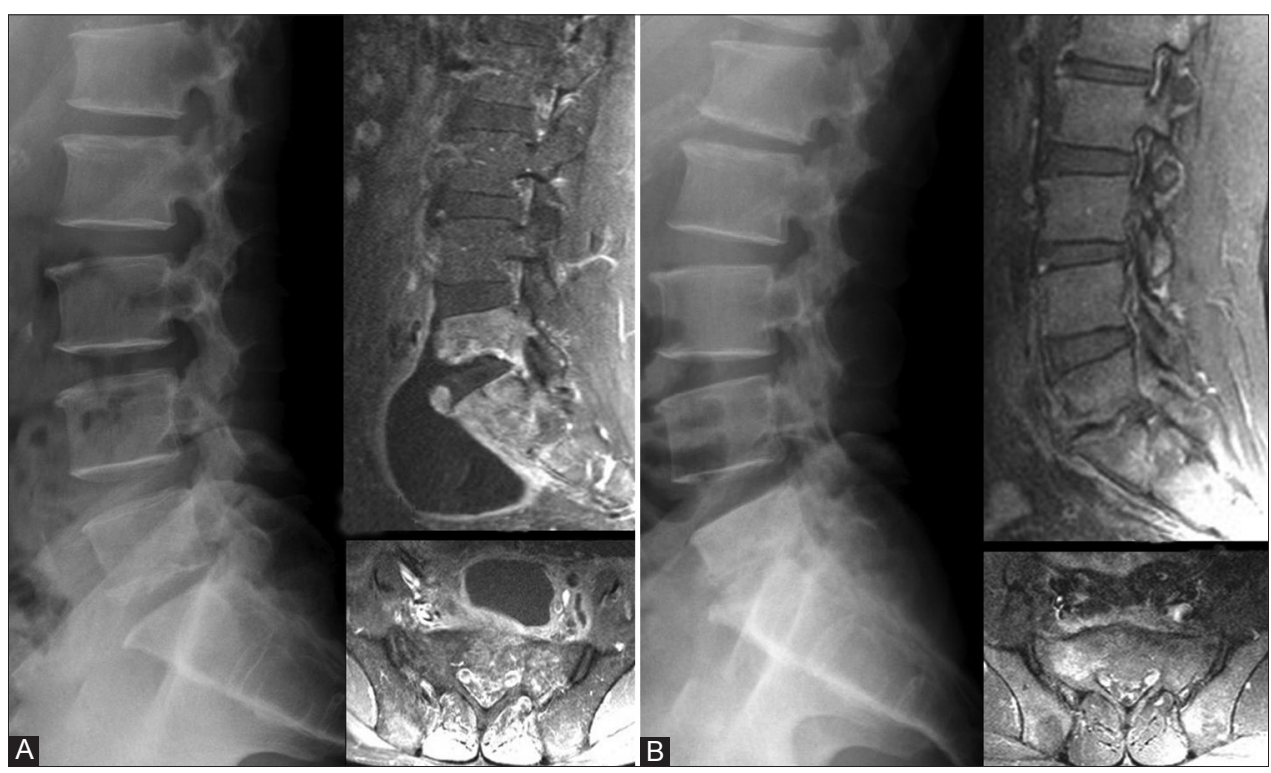

Figure 3: A 57-year-old man with Child's class C liver cirrhosis. The patient had severe low back pain and a grade II bed sore on his buttock. (A) Preoperative lateral radiograph and magnetic resonance (MR) images showing L5-S1 disc and endplate destruction and a large presacral abscess connected with the infected disc level. (B) Follow-up lateral radiograph and MR images at postoperative 9 months showing no presacral abscess and a well-maintained lumbar lordosis. 


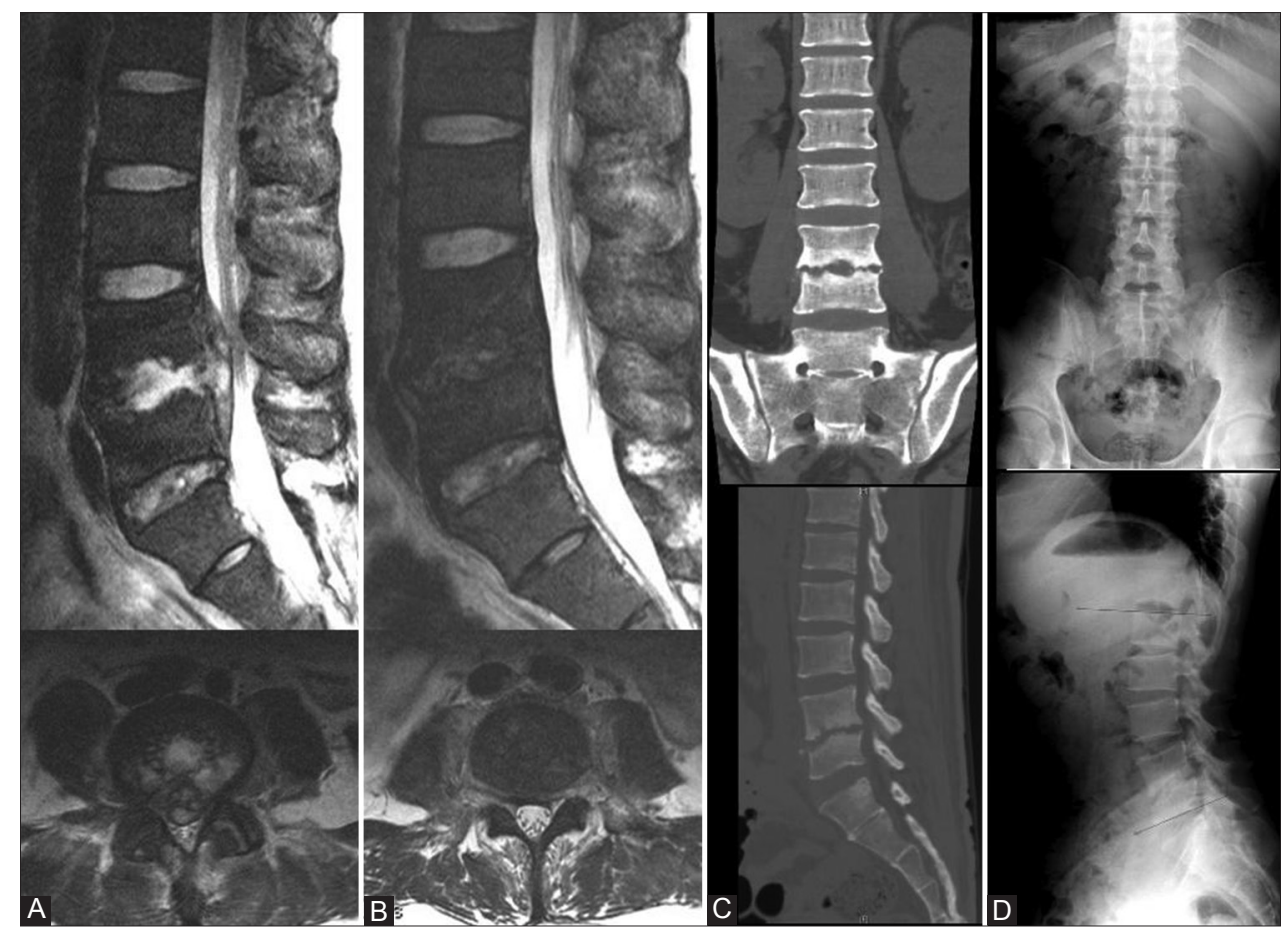

Figure 4: A 32-year-old man presented with severe low back pain and leg pain. His associated medical illness was acute necrotizing pancreatitis with septic shock. (A) Preoperative magnetic resonance (MR) images showing L4-5 disc destruction and epidural abscess formation with nerve compression. (B) Follow-up MR images at postoperative 2 months showing no epidural abscess. (C, D) Follow-up computed tomography (CT) scan images and plain radiographs at 1 year after the surgery showing well-defined vertebral endplates and well-maintained lumbar lordosis, although a slight L4-5 local kyphosis was noted.

PEDD is a superior alternative to CT-guided spinal biopsy and should be considered first for treating infectious lumbar spondylodiscitis.

\section{Efficacy of PEDD for lumbar spondylodiscitis}

Ito et al., used the same posterolateral endoscopic technique to treat 15 patients with pyogenic spondylodiscitis in the thoracic or lumbar spine. They reported that all patients showed immediate back pain reduction after surgery and the infections were successfully treated with subsequent parenteral antibiotic treatment; the average duration of antibiotic therapy was 3.7 weeks. They concluded that posterolateral spinal endoscopic debridement and irrigation resulted in satisfactory clinical results in patients with comorbidities and pyogenic spondylodiscitis. ${ }^{[2]}$

Similarly, we enrolled 14 patients with infectious spondylodiscitis who were treated by PEDD and appropriate parenteral antibiotics. ${ }^{[41]}$ The causative bacteria were identified in $12(85.7 \%)$ of 14 biopsy specimens, thus parenteral antibiotics were administered according to the sensitivity analyses of pathogens. All patients reported immediate back pain relief, with the exception of two patients who required anterior debridement and fusion 1 week and 2 weeks later, respectively. Two other patients had recurrent infection and underwent anterior fusion surgery 1 month and 8 months later, respectively. The remaining 10 patients recovered uneventfully after specific antibiotic therapy. No surgery-related complications or side effects were observed. We concluded that PEDD facilitated the retrieval of adequate specimens and had high diagnostic efficacy, thereby enabling prompt and appropriate antibiotic therapy targeted to the offending pathogens. PEDD is a useful minimally invasive procedure that can be considered as an effective alternative to conventional surgeries for treating uncomplicated spondylodiscitis.

\section{Efficacy of PEDD for complicated infectious spondylodiscitis}

A few reports have described successful utilization of percutaneous drainage and continuous irrigation with a saline-antibiotic solution in the treatment of pyogenic spondylodiscitis accompanied by iliopsoas abscess or marked bone destruction. ${ }^{[36,37]}$ However, this technique lacks endoscopic monitoring for debridement of the lesion site. Furthermore, the continuous irrigation confines the patients to their beds and limits postoperative ambulation and activities.

Two women and four men with poor general health and complicated pyogenic spondylodiscitis who underwent PEDD were evaluated at our institution. The causative bacteria were identified by PEDD in five of six patients. Five patients reported back pain reduction within 1 week 
of surgery and recovered uneventfully after sensitive antibiotic therapy; their CRP values returned to normal ranges within 2-22 weeks. Follow-up MRI studies revealed that the paraspinal abscesses [Figure 3] or epidural abscesses were resolved [Figure 4]. No surgery-related complications were noted during or after the PEDD procedure. ${ }^{[42]} \mathrm{We}$ propose that PEDD is an effective alternative to extensive surgery for complicated infectious spondylodiscitis, particularly in patients with multiple comorbidities.

\section{Conclusions}

With the surgical risks in mind, conservative treatment is the primary therapy for infectious spondylodiscitis, especially in elderly patients and in patients with poor general condition. PED is a minimally invasive procedure and has been reported to result in satisfactory clinical outcomes for lumbar intervertebral disc herniation. We successfully extended the indications of the PED technique in the treatment of spinal infections. Under local anesthesia, PEDD provides retrieval of sufficient specimens for bacteriological diagnosis yielding high positive-culture rates, thereby enabling prompt and sensitive antibiotic therapy targeted to the offending pathogens. The data described herein revealed that this minimal invasive technique could be a good alternative to the traditional anterior open surgery in treating infectious spondylodiscitis. With this percutaneous endoscopic procedure, there is lower morbidity and cost than with open traditional treatment. By selection of proper indications, PEDD could become a routine procedure in the treatment of infectious spondylodiscitis. Based on analytical results, we propose that PEDD is an effective alternative and should be considered prior to extensive anterior surgery for infectious spondylodiscitis, particularly in patients with early-stage spinal infections or serious medical conditions.

\section{REFERENCES}

1. Maiuri F, Iaconetta G, Gallicchio B, Manto A, Briqanti F. Spondylodiscitis. Clinical and magnetic resonance diagnosis. Spine 1997;22:1741-6.

2. Rohde V, Meyer B, Schaller C, Hassler WE. Spondylodiscitis after lumbar discectomy. Incidence and a proposal for prophylaxis. Spine 1998;23:615-20.

3. Gouliouris T, Aliyu SH, Brown NM. Spondylodiscitis: Update on diagnosis and management. J Antimicrob Chemother 2010;65 Suppl 3:11-24.

4. Carragee EJ. Pyogenic vertebral osteomyelitis. J Bone Joint Surg Am 1997;79:874-80.

5. Hadjipavlou AG, Mader JT, Necessary JT, Muffoletto AJ. Hematogenous pyogenic spinal infections and their surgical management. Spine 2000;25:1668-79.

6. Ozuna RM, Delamarter RB. Pyogenic vertebral osteomyelitis and postsurgical disc space infections. Orthop Clin North Am 1996;27:87-94.
7. Emery SE, Chan DP, Woodward HR. Treatment of hematogenous pyogenic vertebral osteomyelitis with anterior debridement and primary bone grafting. Spine 1989;14:284-91.

8. Krodel A, Sturz H, Siebert CH. Indications for and results of operative treatment of spondylitis and spondylodiscitis. Arch Orthop Trauma Surg 1991;110:78-82.

9. Ozuna RM, Delamarter RB. Pyogenic vertebral osteomyelitis and postsurgical disc space infections. Orthop Clin North Am 1996;27:87-94.

10. Rezai AR, Woo HH, Errico TJ, Cooper PR. Contemporary management of spinal osteomyelitis. Neurosurgery 1999;44:1018-25.

11. Carragee EJ. Instrumentation of the infected and unstable spine: A review of 17 cases from the thoracic and lumbar spine with pyogenic infections. J Spinal Disord 1997;10:317-24.

12. An HS, Seldomridge JA. Spinal infections: Diagnostic tests and imaging studies. Clin Orthop Relat Res 2006;444:27-33.

13. Bonfiglio M, Lange TA, Kim YM. The Classic: Pyogenic vertebral osteomyelitis: Disk space infections. 1973. Clin Orthop Relat Res 2006;444:4-8.

14. Chen LH, Fu TS, Kao YH, Tsai TT, Lai PL, Niu CC, et al. Surgical treatment of infectious spondylitis in patients undergoing hemodialysis therapy. Eur Spine J 2010;19:2223-8.

15. Chew FS, Kline MJ. Diagnostic yield of CT-guided percutaneous aspiration procedures in suspected spontaneous infectious diskitis. Radiology 2001;218:211-4.

16. Fouquet B, Goupille P, Jattiot F, Cotty P, Lapierre F, Valat JP, et al. Discitis after lumbar disc surgery. Features of "aseptic" and "septic" forms. Spine 1992;17:356-8.

17. Parker LM, McAfee PC, Fedder IL, Weis JC, Geis WP. Minimally invasive surgical techniques to treat spine infections. Orthop Clin North Am 1996;27:183-99.

18. Rankine JJ, Barron DA, Robinson P, Millner PA, Dickson RA. Therapeutic impact of percutaneous spinal biopsy in spinal infection. Postgrad Med J 2004;80:607-9.

19. Staatz G, Adam GB, Keulers P, Vorwerk D, Gunther RW. Spondylodiskitic abscesses: CT-guided percutaneous catheter drainage. Radiology 1998;208:363-7.

20. Vinicoff PG, Gutschik E, Hansen SE, Karle A, Rieneck K. CT-guided spinal biopsy in spondylodiscitis. Ugeskr Laeger 1998;160:5931-4.

21. Yang SC, Fu TS, Chen LH, Chen WJ, Tu YK. Identifying pathogens of spondylodiscitis: Percutaneous endoscopy or CT-guided biopsy. Clin Orthop Relat Res 2008;466:3086-92.

22. Ito M, Abumi K, Kotani Y, Kadoya K, Minami A. Clinical outcome of posterolateral endoscopic surgery for pyogenic spondylodiscitis: Results of 15 patients with serious comorbid conditions. Spine 2007;32:200-6

23. Nawfel RD, Judy PF, Silverman SG, Hooton S, Tuncali K, Adams DF. Patient and personnel exposure during CT fluoroscopy-guided interventional procedures. Radiology 2000;216:180-4.

24. Paulson EK, Sheafor DH, Enterline DS, McAdams HP, Yoshizumi TT. CT fluoroscopy-guided interventional procedures: Techniques and radiation dose to radiologists. Radiology 2001;220:161-7.

25. Estherhai Jr JL, Gelb I. Adult septic arthritis. Orthop Clin North Am 1991;22:503-14.

26. Lane JG, Falahaee MH, Wojtys EM, Hankin FM, Kaufer H. 
Pyarthrosis of the knee. Treatment considerations. Clin Orthop Relat Res 1990;252:198-204.

27. Ivey M, Clark R. Arthroscopic debridement of the knee for septic arthritis. Clin Orthop Relat Res 1985;199:201-6.

28. Jackson RW. The septic knee arthroscopic treatment. Arthroscopy 1985;1:194-7.

29. Arya S, Crow WN, Hadjipaviou AG, Nauta HJ, Borowski AM, Vierra LA, et al. Percutaneous transpedicular management of discitis. J Vasc Interv Radiol 1996;7:921-7.

30. Crow WN, Borowski AM, Hadjipavlou AG, Walser EM, Arya S, Calme MB, et al. Percutaneous transpedicular automated nucleotomy for debridement of infected discs. J Vasc Interv Radiol 1998;9:161-5.

31. Gebhard JS, Brugman JL. Percutaneous discectomy for the treatment of bacterial discitis. Spine 1994;19:855-7.

32. Hadjipavlou AG, Katonis PK, Gaitanis IN, Muffoletto AJ, Tzermiadianos MN, Crow W. Percutaneous transpedicular discectomy and drainage in pyogenic spondylodiscitis. Eur Spine J 2004;13:707-13.

33. Hanaoka N, Kawasaki Y, Sakai T, Nakamura T, Nanamori K, Nakamura E, et al. Percutaneous drainage and continuous irrigation in patients with severe pyogenic spondylitis, abscess formation, and marked bone destruction. J Neurosurg Spine 2006;4:374-9.

34. Nagata K, Ohashi T, Ariyoshi M, Sonoda K, Imoto H, Inoue A. Percutaneous suction aspiration and drainage for pyogenic spondylitis. Spine 1998;15:1600-6.
35. Onik G, Shang Y, Maroon JC. Automated percutaneous biopsy in postoperative diskitis: A new method. Am J Neuroradiol 1990;11:391-3.

36. Tofuku K, Koga H, Yone K, Komiya S. Continuous irrigation in pyogenic spondylitis accompanied by iliopsoas abscess. Spine 2007;15:E382-7.

37. Yu WY, Siu C, Wing PC, Schweigel JF, Jetha N. Percutaneous suction aspiration for osteomyelitis: Report of two cases. Spine 1991;16:198-202.

38. Yeung AT. The evolution of percutaneous spinal endoscopy and discectomy: State of the art. Mt Sinai J Med 2000;67:327-32.

39. Hermantin FU, Peters T, Quartararo L, Kambin P. A prospective, randomized study comparing the results of open discectomy with those of video-assisted arthroscopic microdiscectomy. J Bone Joint Surg Am 1999;81:958-65.

40. Yeung AT, Tsou PM. Posterolateral endoscopic excision for lumbar disc herniation: Surgical technique, outcome, and complications in 307 consecutive cases. Spine 2002;27:722-31.

41. Yang SC, Fu TS, Chen LH, Niu CC, Lai PL, Chen WJ. Percutaneous endoscopic discectomy and drainage for infectious spondylitis. Int Orthop 2007;31:367-73.

42. Fu TS, Yang SC, Tsai TT, Chen LH, Lai PL, Niu CC, et al. Percutaneous endoscopic debridement and drainage in immunocompromised patients with complicated infectious spondylitis. Minim Invasive Ther Allied Technol 2010;19:42-7. 